\title{
Relação entre Fluência e Compreensão Leitora em Escolares com Dificuldades de Aprendizagem
}

\author{
Vera Lúcia Orlandi Cunha ${ }^{1}$ \\ Maíra Anelli Martins \\ Simone Aparecida Capellini \\ Universidade Estadual Paulista
}

\begin{abstract}
RESUMO - A capacidade para ler e compreender textos é fundamental tanto em nossa vida diária como no contexto educativo. Considerando esse aspecto, o objetivo deste estudo foi comparar a fluência e a compreensão de leitura de escolares do segundo para o quinto ano do ensino fundamental com e sem dificuldades de aprendizagem. Participaram oitenta escolares divididos em oito grupos: GI a GIV (escolares sem dificuldades de aprendizagem); GV a GVIII (escolares com dificuldades de aprendizagem). Por meio da leitura oral de um texto, foram medidos tempo, exatidão, velocidade e compreensão de leitura. Houve diferenças estatisticamente significativas em todas as variáveis, indicando desempenho inferior para os escolares com dificuldades de aprendizagem. Verificou-se que as dificuldades de fluência de leitura interferiram na compreensão leitora.

Palavras-chave: leitura, fluência de leitura, compreensão da leitura, habilidades para leitura
\end{abstract}

\section{Relating between Fluency and Reading Comprehension in Students with Learning Difficulties}

\begin{abstract}
The capacity to read and comprehending text is essential in our daily life and in the educational context. Considering this aspect the objective of this study was to compare fluency and reading comprehension of students from second to fifth grade of primary school with and without learning difficulties. The eighty students who participated were divided into eight groups: GI to GIV (students without learning difficulties); GV to GVIII (students with learning difficulties). Of the oral reading of a text were measured the time, accuracy, speed and reading comprehension. Statistically significant differences were observed on all these variables indicating inferior performance to the students with learning difficulties. It was found that difficulties in reading fluency interfered with reading comprehension.
\end{abstract}

Keywords: reading, reading fluency, reading comprehension, reading skills

O ingresso no processo de escolarização requer uma série de habilidades e competências que se constituem como pré-requisitos para as aprendizagens que se processarão. Entretanto, é muito comum que escolares enfrentem problemas de diversas ordens nos primeiros anos de escolarização, uma vez que existe, nesse período, uma gama de fatores de ordens interna e externa, tanto próprias do indivíduo quanto da escola ou do ambiente, capazes de interferir na aprendizagem e constituírem obstáculos à integração do pensar, sentir, falar, ouvir e agir (Tonelloto \& Gonçalves, 2002).

Os problemas apresentados pelos escolares durante a escolarização puderam ser observados por meio dos dados divulgados pelo Programa Internacional de Avaliação de Alunos (Organização para Cooperação e Desenvolvimento Econômico [OCDE], 2014), que mede o conhecimento dos alunos de quinze e dezesseis anos de idade em três matérias: matemática, ciências e leitura. Os dados revelaram que o Brasil, entre 64 países, ficou na $55^{\circ}$ colocação no ranking da leitura, tendo obtido 410 pontos em leitura, $391 \mathrm{em}$ matemática e 405 em ciência.

Em 2012, cerca de metade $(49,2 \%)$ dos escolares no Brasil desempenharam abaixo do nível básico de proficiência

1 Endereço para correspondência: Rua Vereador Clovis Camargo Bueno, n 218, Bairro Jardim das Flores, Palmital, SP, Brasil. CEP: 19.970-000. E-mail:verafono@uol.com.br
(Nível 2), o que indica que esses escolares são capazes de encontrar informações explícitas nos textos e relacioná-las com o cotidiano deles apenas, obtendo somente o grau mínimo de habilidade de leitura. Observa-se, portanto, no panorama da realidade educacional brasileira, a existência de um número crescente de escolares com dificuldades que não conseguem acompanhar as atividades de leitura no contexto escolar (OCDE, 2014).

A literatura define a dificuldade de aprendizagem como sendo transitória (Rebelo, 1993; Capellini, 2004, Capellini, Castro Silva, Silva, \& Pinheiro, 2009; Sampaio, Pinheiro, \& Silva, 2011). A dificuldade de aprendizagem caracteriza-se por um grupo heterogêneo de manifestações que ocasiona baixo rendimento em leitura, escrita e cálculo-matemático. As dificuldades de aprendizagem fazem com que os escolares não consigam acompanhar as atividades de leitura e escrita no contexto escolar principalmente por motivos relacionados ao sujeito que aprende ou ao professor, aos conteúdos pedagógicos, à falta de oportunidades para a aprendizagem, a condições socioeconômicas desfavoráveis e a propostas/ métodos de ensino que não sejam adequadas ao perfil e a necessidade desses escolares. Ou seja, eles sofrem as consequências de fatores extrínsecos diretamente na sua aprendizagem, tendo, muitas vezes, o fracasso acadêmico como resultado final (Capellini et al., 2009; Zorzi, 2008). 
A todo esse contexto do escolar com dificuldade de aprendizagem, adiciona-se o fato de a aquisição e o desenvolvimento da leitura apresentar-se como uma atividade complexa, pois é uma habilidade composta por múltiplos processos interdependentes (Salles \& Parente, 2004). Entre esses processos estão a codificação semântica, a aquisição de vocabulário, a compreensão das ideias do texto e a criação de modelos mentais do texto, processos estes que levam ao objetivo primeiro da leitura: a compreensão da mensagem escrita.

Por ser a compreensão primordial na função da leitura, Stothard (2004) argumentou que é de conhecimento amplo que os problemas no processo da leitura constituem um obstáculo à aprendizagem, pois todas as disciplinas curriculares requerem que os escolares leiam e extraiam as informações importantes de que precisam para seu aprendizado escolar. Sendo assim, aqueles escolares com problemas de compreensão não conseguem realizar essas tarefas e ficam atrasados em relação aos seus colegas de classe. De acordo com Stothard, aproximadamente um entre dez escolares experimenta problemas específicos de compreensão da leitura. Tais dificuldades variam de 07 a 26 meses de atraso nas habilidades de compreensão.

Essas dificuldades podem ser encontradas tanto na aprendizagem de capacidades elementares do desenvolvimento da leitura como na decodificação fonológica, ou seja, na habilidade de conversão letra-som. Segundo Calhoon (2005), essas dificuldades impedem que o escolar desenvolva outras habilidades, como o domínio da linguagem, o crescimento do vocabulário, a escrita e o conhecimento das palavras, fato que repercutirá no processo de compreensão da mensagem escrita e, consequentemente, no desenvolvimento de aprendizagens posteriores.

Em estudo realizado por Zucoloto e Sisto (2002), os participantes apresentaram baixa compreensão da leitura. Esse fato, de acordo com os autores, pode ter ocorrido pela ação de diferentes fatores, que podem ter atuado de forma conjunta ou isolada: dificuldade em diferenciar a forma visual-ortográfica dos signos escritos, dificuldade para processar a ordem sequencial dos estímulos, ênfase na decodificação em detrimento da integração do significado, dificuldade para criar um significado global e ordenar a informação segundo a lógica organizacional do texto, bem como dificuldades que podem ser oriundas de problemas de vocabulário.

Dessa forma, vários fatores podem contribuir para dificuldades nesse processo, uma vez que a habilidade de leitura envolve componentes desde a decodificação até a compreensão. A decodificação refere-se aos processos de reconhecimento da palavra escrita, que, de acordo com Sánchez, García e Gonzalez (2007), é a competência aparentemente mais elementar de todas, a qual se refere a um processo que permite transformar os signos ortográficos das palavras escritas em linguagem. A compreensão, por sua vez, é definida como o processo pelo qual as palavras, sentenças ou textos são interpretados.

Durante o processo da aprendizagem da leitura, a criança aprende a associar a forma ortográfica da palavra à sua forma fonológica atribuindo, por conseguinte, significado a essa palavra. Portanto, o processo de aquisição está relacionado ao reconhecimento e à compreensão dessas palavras (Batista \& Fusco, 2012). Assim sendo, a apropriada decodificação pode garantir a compreensão do material lido, pois a transformação automática do código escrito em código oral promove a adequação da velocidade, da acurácia e de uma apropriada prosódia, o que possibilita a leitura precisa do texto, fazendo com que a atenção seja direcionada para os processos da compreensão (Penna, Sabaté, \& Burin, 2014). Por conseguinte, essa relação entre leitura de palavras isoladas e compreensão de leitura ocorre porque, quanto maior a rapidez na identificação de palavras, maior é a capacidade que a memória de trabalho tem para se dedicar às operações consideradas de alto nível como a análise sintática, a integração semântica dos constituintes da frase e a integração das frases na organização textual, levando à compreensão da leitura (Akyol, \& Ulusoy, 2010; Cunha, Oliveira, \& Capellini, 2010; Morais, 1996; Salles, \& Parente, 2002, 2004; Sánchez et al., 2007).

Para Ávila, Carvalho e Kida (2009), com a progressão da automaticidade de elementos envolvidos no reconhecimento de palavras e com o aumento da velocidade na decodificação e no reconhecimento de letras e palavras, a leitura torna-se progressivamente mais rápida, precisa e fluente. Para as autoras, a fluência interfere na capacidade dos escolares para a compreensão da leitura, pois, quanto mais automáticos forem os processos fundamentais de decodificação e reconhecimento da palavra, mais rápida e precisa se torna a leitura (portanto, mais fluente), o que ocasionará uma maior disponibilidade da atenção e da memória para processos de alta ordem, melhorando, portanto, o nível de sua compreensão (Bell, 2001; Costa, 2004; Ferreira, 2009; Fuchs, Fuchs Douglas, Hosp, \& Jenkins, 2001; O'connor, Swanson, \& Geraghty, 2010; Snellings, van der Leij, de Jong, \& Blok, 2009).

O processo de leitura que ocorre no sistema de escrita alfabético pode ser realizado, pelo menos, de duas maneiras: por meio de um processo visual direto (rota lexical) ou de um processo envolvendo mediação fonológica (rota fonológica). A rota fonológica utiliza o processo de conversão grafemafonema, traduzindo letras ou grupos de letras em fonemas, ou seja, as representações fonêmicas armazenadas ativam as formas fonológicas das palavras, que, por sua vez, levam à ativação das representações semânticas e ortográficas correspondentes (Morais, 1996; Pinheiro, 1994).

Na leitura pela rota lexical, as representações de palavras familiares são armazenadas em um léxico de entrada visual, que é ativado pela apresentação visual de uma palavra, seguido pela obtenção do significado a partir do sistema semântico (conhecimento sobre os significados de palavras familiares) e, então, a palavra pode ser articulada. No processo de identificação de palavras, o uso da rota lexical permite acesso mais rápido ao léxico mental por ser um procedimento de acesso direto ao significado a partir da estrutura gráfica. Ao contrário, a rota fonológica é um procedimento sequencial, ao menos no início do desenvolvimento da leitura, e, portanto, mais lento que o anterior (Salles \& Parente, 2002, 2004).

A decodificação tem, assim, grande interferência e influência no processo de compreensão de leitura. Assim, é essencial que o escolar possua uma boa decodificação para ter uma leitura fluente. No entanto, isso não garante que ele 


\section{Participantes}

tenha uma compreensão de todas as informações contidas no texto (Cunha \& Capellini, 2009a, 2009b; Giangiacomo \& Navas, 2008; Manyak \& Bauer, 2008; Mcquiston, O’shea, \& Mccollin, 2008; Salgado \& Capellini, 2008; Wise, Seycik, Morris, Lovett, \& Wolf, 2007). Isso ocorre devido ao fato de a compreensão leitora incluir vários processos cognitivos interrelacionados. Entre eles, os processos básicos de leitura, que são o reconhecimento e a extração do significado das palavras impressas — que são requisitos necessários, porém não são suficientes. Isso porque a compreensão da leitura depende da ativação de relevantes conhecimentos que estão fortemente relacionados com o desenvolvimento do vocabulário, da linguagem oral, de habilidades linguísticas, habilidades de memória, capacidade de realizar inferências e da experiência de mundo de cada indivíduo, além do desenvolvimento da expressão clara e organizada de ideias, tanto na forma oral como na escrita (Braibant, 1997; Santos, 2009).

De acordo com Sánchez (2008), sabe-se que, ao final da educação dos primeiros anos, a velocidade com que os escolares leem as palavras prediz seu nível de compreensão. Dessa maneira, ao se comparar dois escolares com os mesmos conhecimentos prévios, a mesma capacidade de memória de trabalho e a mesma habilidade para operar com os marcadores discursivos, se um deles lê mais rápido, provavelmente compreenderá o texto um pouco melhor. Naturalmente, quem compreende um pouco melhor tem mais probabilidades de envolver-se em mais experiências de leituras e isso, ainda, ocasionará uma leitura automática mais eficaz, que aumentará as diferenças prévias dos escolares. Essas diferenças, que vão se produzindo entre os escolares a respeito de qualquer das habilidades implicadas (inclusive as mais elementares), repercutem nas obtenções globais posteriores. Isso foi demonstrado em estudos realizados em diferentes níveis acadêmicos que indicaram que as dificuldades de compreensão de leitura que aparecem nos primeiros anos no ensino fundamental podem prejudicar o escolar em toda sua vida acadêmica. Os estudos de Guidetti e Martinelli (2007) e de Oliveira, Boruchovitch e Santos (2009) apontaram para as dificuldades dos escolares do ensino fundamental. Oliveira, Catalice e Freitas (2009) investigaram as dificuldades de escolares do ensino médio, enquanto Oliveira e Santos (2006) trabalharam com escolares de ensino superior e consideraram que a habilidade de compreensão em leitura no ensino superior pode ser a diferença entre um aprendizado técnico científico de qualidade ou não.

Considerando os aspectos apresentados, este estudo teve como objetivo descrever e comparar o desempenho em fluência de leitura e compreensão de leitura de escolares do segundo ao quinto ano do ensino fundamental com e sem queixas de dificuldades de aprendizagem.

\section{Método}

Este estudo foi submetido ao Comitê de Ética em Pesquisa da FFC/UNESP - Marília-SP e aprovado sob o Protocolo $n^{\circ}$ $1881 / 2008$.
Foram avaliados 80 escolares do segundo ao quinto ano do Ensino Fundamental, de ambos os gêneros, na faixa etária de sete a dez anos de idade, de uma escola municipal da cidade de Marília-SP, divididos em oito grupos. Quatro grupos não apresentavam queixas de dificuldades de aprendizagem constantes em prontuário escolar enquanto quatro as apresentavam. Os escolares foram selecionados pelos professores com base no desempenho acadêmico em dois bimestres consecutivos, sendo considerados escolares com dificuldades de aprendizagem aqueles que apresentavam desempenho insuficiente (de acordo com a média escolar em provas de língua portuguesa) e sem dificuldades de aprendizagem aqueles que apresentavam desempenho suficiente (de acordo com a média escolar em provas de língua portuguesa).

Os critérios de inclusão para a seleção da amostra foram a assinatura do Termo de Consentimento Livre e Esclarecido pelos pais ou responsáveis pelos escolares; escolares com acuidade visual, auditiva e desempenho cognitivo dentro dos padrões da normalidade, conforme descrição no prontuário escolar e relato dos professores. Os critérios de exclusão para a seleção da amostra foram a presença de deficiência sensorial (visual e auditiva), motora ou cognitiva nos escolares matriculados na escola, conforme descrição no prontuário escolar e relato dos professores. De acordo com a Política Nacional de Saúde da Pessoa Portadora de Deficiência, a pessoa com deficiência é aquela que pode apresentar deficiência mental, motora, sensorial e/ou múltipla. No que concerne à deficiência motora, refere-se a uma variedade de condições neurossensoriais que afetam mobilidade, coordenação motora geral ou de fala. Foi critério de exclusão também a presença de síndromes genéticas ou neurológicas.

O Termo de Consentimento Livre e Esclarecido foi entregue aos pais ou responsáveis após esclarecimentos sobre a pesquisa. Após a sua devolução com assinatura de consentimento para a participação, foram formados grupos compostos cada um por 10 escolares indicados pelos professores das respectivas séries escolares, da seguinte maneira:

- Grupos GI, GII, GIII e GIV - compostos respectivamente pelos escolares do $2^{\circ}, 3^{\circ}, 4^{\circ}$ e $5^{\circ}$ ano sem queixas de dificuldades de aprendizagem.

- Grupos GV, GVI, GVII e GVIII - compostos respectivamente pelos escolares do $2^{\circ}, 3^{\circ}, 4^{\circ}$ e $5^{\circ}$ ano com queixas de dificuldades de aprendizagem.

\section{Instrumentos}

Foram utilizados textos do gênero narrativo, por ser um gênero ao qual os escolares estão mais expostos, de acordo com levantamento nos livros didáticos do $2^{\circ}$ ao $5^{\circ}$ utilizados na rede pública de Ensino Fundamental no município onde a pesquisa foi realizada. Os textos narrativos dos livros didáticos de cada série escolar foram inicialmente selecionados pelos professores de cada série de quatro escolas municipais. Os textos que receberam maior número de indicações foram encaminhados para uma nova seleção, realizada 
agora por uma banca de profissionais (fonoaudiólogos, psicopedagogos, pedagogos e coordenadores pedagógicos de escolas do Ensino Fundamental), que deveriam indicar a escolha dos textos de acordo com a representatividade (os juízes deveriam qualificar a representatividade considerando: demarcação clara das ideias do texto, identificação da ideia global, integração das informações do texto, coesão e coerência entre as informações contidas no texto, possibilidade de integrar as informações contidas no texto com conhecimentos já adquiridos) e de acordo com a dificuldade de cada texto para cada série escolar (considerando: a extensão do texto, ou seja, número de palavras, vocabulário utilizado, adequação do tema à idade e à escolaridade, ortografia e estrutura sintática adequada à idade e à escolaridade). Essas pontuações foram realizadas seguindo escala de Likert. Foi atribuída a pontuação de 1 a 4 para cada título do texto, indicando qual era considerado mais adequado segundo a representatividade e a dificuldade de cada um deles, de acordo com cada ano escolar.

As perguntas para a verificação da compreensão do texto lido foram elaboradas de acordo com o substrato teórico do Modelo de Representação Mental de Compreensão de Leitura, desenvolvido por Kintsch e Van Dijk (1978). Segundo esse modelo, a habilidade de leitura se suporta em compreensão literal e inferencial, baseando-se na macro e microestrutura do texto. Dessa forma, para a elaboração das perguntas, realizou-se inicialmente a análise das proposições (ou ideias) que estão explícitas ou implícitas no texto, ou seja, verificando quais são as informações literais ou inferenciais contidas, assim como as relações causais entre essas proposições. Essa análise foi realizada em cada tipo de texto selecionado. Assim, seguindo esses critérios, as perguntas foram elaboradas de acordo com dois grupos de classificação, sendo duas perguntas literais e duas inferenciais, que foram, depois, subdivididas em quatros subgrupos, sendo uma pergunta literal relacionada à microestrutura do texto e uma literal relacionada à macroestrutura, e outros dois subgrupos compostos de uma pergunta inferencial relacionada à microestrutura e outra inferencial relacionada à macroestrutura, chegando-se a uma composição final de quatro perguntas. $\mathrm{O}$ julgamento da adequação das questões quanto à qualidade, assim como a classificação do tipo de pergunta foi realizado por três juízes (um fonoaudiólogo, um psicólogo e um psicopedagogo).

\section{Procedimentos}

A coleta de dados com a leitura oral dos escolares foi realizada na própria escola, individualmente, no horário de aula. As perguntas foram realizadas de forma oral pela pesquisadora e as respostas dos escolares foram gravadas para análise posterior, com o objetivo de que possíveis dificuldades na produção escrita não interferissem no desempenho das respostas.

O tempo de leitura foi cronometrado e marcado em segundos e as palavras lidas incorretamente anotadas. Também foi calculada a velocidade de leitura de acordo com Ramos (2005): multiplicando-se o número de palavras do texto por 60 segundos, e esse valor dividido pelo tempo total da leitura em segundos, conforme a seguinte fórmula, obtendo-se o número de palavras lidas por minuto:

Velocidade de leitura $=\underline{\text { número de palavras do texto x } 60 \text { segundos }}$ tempo total de leitura do texto

O desempenho em compreensão de leitura foi avaliado de acordo com o número de respostas corretas apresentado pelo escolar segundo critérios baseados no domínio da habilidade de compreensão do texto lido estabelecidos pelos pesquisadores deste estudo. Quando o escolar respondia a todas as questões, tinha $100 \%$ de compreensão; a três perguntas, 75\% de compreensão; a 2 perguntas, 50\%; a 1 pergunta, $25 \%$ de compreensão; a nenhuma pergunta, $0 \%$ de compreensão do texto.

\section{Resultados}

A análise dos resultados foi realizada pelo programa SPSS (Statistical Package for Social Sciences), em sua versão 20.0. Foi adotado o nível de significância de 5\% $(0,050)$.

Foi aplicado o Teste de Mann-Whitney, com o intuito de verificar possíveis diferenças na comparação entre os grupos sem dificuldade (do $2^{\circ}$ ao $5^{\circ}$ ano - de GI a GIV) e os grupos com dificuldade (do $2^{\circ}$ ao $5^{\circ}$ ano - de GV a GVIII) em cada série nas variáveis Tempo Total de Leitura (TTL), Palavras Lidas Incorretamente (PLI), Velocidade de Leitura (VL), Compreensão de Leitura (CL).

A Tabela 1 mostra a comparação entre os grupos GI e $\mathrm{GV}$ em todas as variáveis. Observa-se que, nesses grupos, houve diferença estatisticamente significante em PLI e $\mathrm{CL}$, indicando que as diferenças dos desempenhos entre os grupos nessas variáveis foram superiores às demais. Para PLI, o desempenho do grupo GV foi inferior em relação ao desempenho do grupo GI, pois o GV apresentou média superior, indicando que, no grupo com dificuldades de leitura, houve maior número de palavras lidas incorretamente. $\mathrm{O}$ mesmo ocorreu para CL, com a média do desempenho para o grupo GV inferior ao grupo GI, indicando que o $\mathrm{GV}$ apresentou desempenho inferior em relação ao GI.

A Tabela 2 mostra a comparação entre os grupos GII e GVI em todas as variáveis. Observa-se que, nesses grupos, houve diferença estatisticamente significante em todas as

Tabela 1. Comparação entre os grupos GI e GV em todas as variáveis

\begin{tabular}{lccccc}
\hline Variável & Grupo & $\boldsymbol{n}$ & $\boldsymbol{M}$ & $\boldsymbol{D P}$ & Sig. $(\boldsymbol{p})$ \\
\hline TTL & I & 10 & 143,30 & 84,09 & 0,059 \\
& V & 10 & 232,00 & 106,02 & \\
PLI & I & 10 & 8,80 & 7,83 & $0,026^{*}$ \\
& V & 10 & 15,90 & 6,42 & \\
VL & I & 10 & 40,64 & 21,04 & 0,112 \\
& V & 10 & 26,97 & 13,05 & \\
CL & I & 10 & 67,50 & 31,29 & $0,030^{*}$ \\
& V & 10 & 35,00 & 26,87 & \\
\hline
\end{tabular}

Nota. TTL (tempo total de leitura, marcado em segundos); PLI (número de palavras lidas incorretamente); VL (velocidade de leitura, marcada em segundos); CL (compreensão de leitura, média das perguntas respondidas corretamente). 
variáveis, ou seja, em TTL, PLI, VL e CL, indicando que o desempenho do grupo GVI, grupo com dificuldades de leitura, foi inferior em relação ao grupo GII em todas as variáveis.

Tabela 2. Comparação entre os grupos GII e GVI em todas as variáveis

\begin{tabular}{lccccc}
\hline Variável & Grupo & $\boldsymbol{n}$ & $\boldsymbol{M}$ & $\boldsymbol{D P}$ & Sig. $(\boldsymbol{p})$ \\
\hline TTL & II & 10 & 206,00 & 83,11 & $0,041^{*}$ \\
& VI & 10 & 459,30 & 429,51 & \\
PLI & II & 10 & 9,80 & 5,65 & $0,002 *$ \\
& VI & 10 & 50,50 & 52,25 & \\
VL & II & 10 & 75,13 & 26,91 & $0,002 *$ \\
& VI & 10 & 30,50 & 21,97 & \\
CL & II & 10 & 75,00 & 31,18 & $0,003 *$ \\
& VI & 10 & 22,50 & 32,17 & \\
\hline
\end{tabular}

Nota. TTL (tempo total de leitura, marcado em segundos); PLI (número de palavras lidas incorretamente); VL (velocidade de leitura, marcada em segundos); CL (compreensão de leitura, média das perguntas respondidas corretamente)

Já na Tabela 3, observa-se a comparação entre os grupos GIII e GVII, sendo que, nesses grupos, também ocorreu diferença estatisticamente significante em todas as variáveis, ou seja, em TTL, PLI, VL e CL, indicando que o desempenho do grupo GVII, grupo com dificuldades de leitura, foi inferior em relação ao grupo GIII em todas as variáveis.

Tabela 3. Comparação entre os grupos GIII e GVII em todas as variáveis

\begin{tabular}{lccccc}
\hline Variável & Grupo & $\boldsymbol{n}$ & $\boldsymbol{M}$ & $\boldsymbol{D P}$ & Sig. $(\boldsymbol{p})$ \\
\hline TTL & III & 10 & 168,40 & 46,10 & $0,008^{*}$ \\
& VII & 10 & 392,70 & 377,40 & \\
PLI & III & 10 & 6,20 & 3,80 & $0,000^{*}$ \\
& VII & 10 & 36,20 & 30,56 & \\
VL & III & 10 & 98,35 & 21,68 & $0,006^{*}$ \\
& VII & 10 & 61,68 & 30,07 & \\
CL & III & 10 & 85,00 & 26,87 & $0,034^{*}$ \\
& VII & 10 & 55,00 & 34,96 & \\
\hline
\end{tabular}

Nota. TTL (tempo total de leitura, marcado em segundos); PLI (número de palavras lidas incorretamente); VL (velocidade de leitura, marcada em segundos); CL (compreensão de leitura, média das perguntas respondidas corretamente)

A Tabela 4 mostra a comparação entre os grupos GIV e GVIII em todas as variáveis. Observa-se que nesses grupos houve diferença estatisticamente significante em TTL, VL e CL, indicando que o desempenho do grupo GVIII, com dificuldade de aprendizagem, foi inferior em relação ao grupo GIV.

\section{Discussão}

$\mathrm{Na}$ comparação entre os grupos GI e GV, grupos formados pelos escolares do $2^{\circ}$ ano, respectivamente sem e com queixas de dificuldade de aprendizagem, observou-se que houve diferença estatisticamente significante para PLI e CL, indicando desempenho superior para o grupo GI, sem
Tabela 4. Comparação entre os grupos GIV e GVIII em todas as variáveis

\begin{tabular}{lccccc}
\hline Variável & Grupo & $\boldsymbol{n}$ & $\boldsymbol{M}$ & $\boldsymbol{D P}$ & Sig. $(\boldsymbol{p})$ \\
\hline TTL & IV & 10 & 210,50 & 57,81 & $0,002^{*}$ \\
& VIII & 10 & 439,70 & 195,83 & \\
PLI & IV & 10 & 15,30 & 8,542 & 0,211 \\
& VIII & 10 & 26,30 & 2,60 & \\
VL & IV & 10 & 78,98 & 21,00 & $0,001^{*}$ \\
& VIII & 10 & 40,24 & 16,17 & \\
CL & IV & 10 & 77,50 & 24,86 & $0,003^{*}$ \\
& VIII & 10 & 37,50 & 21,25 & \\
\hline
\end{tabular}

Nota. TTL (tempo total de leitura, marcado em segundos); PLI (número de palavras lidas incorretamente); VL (velocidade de leitura, marcada em segundos); CL (compreensão de leitura, média das perguntas respondidas corretamente)

dificuldade de aprendizagem. Os resultados indicaram que a compreensão de leitura para o GI foi superior ao GV e que os escolares do primeiro grupo apresentaram uma leitura mais precisa, o que pode ter influenciado seu desempenho na compreensão final do texto, concordando com os estudos anteriores, que sugeriram uma relação entre automatismo (precisão e rapidez) no reconhecimento de palavras e na compreensão de leitura, pois, quanto mais rápida e precisa for a identificação de cada palavra, maior a capacidade da memória de trabalho consagrada às operações de análise sintática, de integração semântica dos constituintes da frase e de integração das frases na organização textual, processos importantes para a compreensão da leitura, uma vez que o acesso preciso e rápido (automático) ao léxico mental influencia o tempo gasto na leitura de um texto e, por conseguinte, a compreensão final da mensagem escrita (Akyol \& Ulusoy, 2010; Cunha et al., 2010; Morais, 1996; Salles \& Parente, 2002, 2004; Zucoloto \& Sisto, 2002).

Desse modo, nossos resultados corroboram também os estudos de Batista e Fusco (2012), assim como os de Penna et al. (2014), que mostraram que decodificação precisa pode garantir a compreensão do material lido, pois a transformação automática do código escrito em código oral promove a adequação da velocidade, da acurácia e de uma prosódia apropriada, o que possibilita que a atenção seja direcionada para os processos da compreensão. Assim, os escolares do segundo ano sem dificuldades de leitura, pertencentes ao GI, tiveram um desempenho similar aos escolares com dificuldades do grupo GIV, também do segundo ano, por estarem ainda em processo de aprendizagem, portanto, sem terem adquirido ainda o automatismo na decodificação fazendo com que seus desempenhos sejam semelhantes.

Nos desempenhos dos grupos GII e GVI (terceiro ano) e GIII e GVII (quarto ano), observaram-se diferenças estatisticamente significante em todas as variáveis, ou seja, para TTL, PLI, VL e CL, sugerindo que, com a seriação, os escolares sem dificuldades vão tendo seu desempenho superior a cada ano. $\mathrm{O}$ mesmo não ocorre para os grupos com dificuldade de aprendizagem, fazendo com que aumentem as diferenças dos seus desempenhos em relação aos escolares sem dificuldades. Esses dados sugerem que as habilidades de leitura, como a decodificação precisa e rápida e a compreensão para os grupos sem dificuldade, ocasionam 
diferenças superiores aos seus desempenhos quando comparados aos grupos com dificuldade de aprendizagem.

Tais diferenças podem ser devidas ao uso das rotas de leitura utilizadas de forma distinta por esses grupos. A rota fonológica é mais utilizada pelos leitores pouco habilidosos ou em início do processo de aprendizagem de leitura (Salles \& Parente, 2002, 2004), o que indica que os leitores com dificuldades de leitura utilizaram essa rota, fazendo a relação grafema-fonema na decodificação das palavras, concordando com os estudos de Silva e Capellini (2010) e de Snellings et al. (2009). Talvez por ser uma rota mais lenta de acesso ao léxico, influenciando habilidades de leitura como a velocidade e precisão, e, consequentemente, requerer maior carga de memória de trabalho e atenção consciente nesse processo mais básico, o uso dessa estratégia permitiria a interferência na compreensão da história, modificando seu significado. Por outro lado, os grupos sem dificuldade de aprendizagem podem ter utilizado tanto a rota lexical quanto a fonológica, melhorando também a precisão de leitura, sendo tais habilidades próprias dos leitores habilidosos, o que levaria a uma maior velocidade e precisão de leitura textual e, como consequência, uma melhor compreensão de leitura, resultando em um desempenho final superior das habilidades de leitura, fato que corrobora estudos anteriores (Breznitz, 2006; Eckert, Ardoin, Daly, \& Martens, 2002; Morais, 1996; Perfetti, 1985; Saine, Lekkanen, Ahonen, Tovlanen, \& Lyytinen, 2010; Salles \& Parente, 2002, 2004).

Na comparação entre os grupos GIV e GVIII (quinto ano), houve diferença estatisticamente significante em TTL, VL e CL, com desempenho superior para o grupo sem dificuldade. Esses resultados sugerem que os desempenhos no tempo total e na velocidade de leitura podem ter influenciado o desempenho em compreensão, indicando que a velocidade mais lenta de leitura, que ocasionou um tempo total de leitura maior para o grupo com dificuldade de aprendizagem, GVIII, pode ter provocado um desempenho inferior em compreensão. Tais dados corroboraram estudos de Salles e Parente $(2002,2004)$, que indicaram que a velocidade de leitura e o tempo de leitura de palavras correlacionaram-se significantemente com habilidade de compreensão de leitura textual. Estão de acordo também com Sanchéz (2008), que argumentou que, ao final da educação dos primeiros anos, a velocidade com que os escolares leem as palavras prediz seu nível de compreensão.

Para este estudo, assumem-se as proposições de Ávila et al. (2009), Bell (2001), Costa (2004), Ferreira (2009), Fuchs et al. (2001), O’connor et al. (2010) e Snellings et al. (2009), segundo os quais a velocidade de leitura tem demonstrado ser uma medida capaz e eficiente para predizer a fluência, como um reflexo do aumento na velocidade da decodificação, do reconhecimento de letras e palavras, podendo ser utilizada para o monitoramento da compreensão de leitura, principalmente nos anos iniciais. Tal fato se justifica, pois, à medida que os escolares avançam em sua aprendizagem, vão tornando-se leitores mais qualificados.

Dessa forma, nossos resultados corroboraram Sánchez (2008), que argumentou que a diferença da habilidade de decodificação entre os escolares influencia a compreensão, aumentando as diferenças entre os escolares, conforme verificado em nossos achados. Essas diferenças entre os escolares a respeito de qualquer das habilidades envolvidas repercutem nas obtenções globais posteriores, aumentando cada vez mais a diferenças entre os escolares, conforme verificado nos estudos de Guidetti e Martinelli (2007), Oliveira, Boruchotvitch e Santos (2009), Oliveira, Cantalice e Freitas (2009) e de Oliveira e Santos (2006).

\section{Conclusão}

Na comparação entre os grupos com e sem dificuldade de aprendizagem, observaram-se diferenças estatisticamente significantes. Os dados sugerem que os desempenhos tornaram-se superiores nos grupos de maior grau escolar, indicando que, com o aumento da escolaridade, os grupos sem dificuldade de aprendizagem têm seus desempenhos cada vez superiores, enquanto que, com os grupos com dificuldade de aprendizagem, ocorre o inverso, ou seja, seus desempenhos vão se tornando inferiores. Esses dados sugerem que, à medida que ocorre aumento da quantidade e da dificuldade dos textos para leitura em sala de aula, os escolares com dificuldade de aprendizagem vão apresentando seu desempenho cada vez mais defasado em relação aos escolares sem dificuldade de aprendizagem, pois estes conseguem abstrair pela leitura dos textos as informações necessárias para sua aprendizagem. Dessa forma, constatouse que habilidades como a decodificação influenciam a velocidade e o tempo total de leitura. Por isso, deveriam ter sido trabalhadas desde a pré-escola, com o objetivo de prevenir possíveis dificuldades na fluência, e nas habilidades que interferem no processo final da compreensão para a obtenção do sentido global da mensagem escrita.

Atualmente, ainda se observa certa resistência em aceitar a importância da decodificação e fluência de leitura para o futuro desempenho acadêmico do leitor. Toda a sua aprendizagem depende da leitura e esta, por sua vez, depende do conjunto de habilidades para uma compreensão profunda. Espera-se que, com os resultados obtidos neste e em outros estudos sobre o tema, possam consolidar-se as bases de comprovações científicas sobre a hipótese levantada e esclarecer-se/demonstrar-se aos professores e profissionais da área da educação o quão necessário é se trabalhar com a decodificação e fluência da leitura dentro da sala de aula para prevenir possíveis dificuldades no ensino da leitura e no aprendizado acadêmico.

\section{Referências}

Akyol, H., \& Ulusoy, M. (2010). Pre-service teachers' use of reading strategies in their own readings and future classrooms. Teaching and Teacher Education, 26, 878-884. doi: 10.1016/j. tate.2009.10.026

Ávila, C. R. B., Carvalho, C. A. F., \& Kida, A. S. B. (2009). Parâmetros de fluência e compreensão de leitura. In T. Barbosa et al. (Eds.), Temas em dislexia (pp.103-113). São Paulo: Artes Médicas. 
Batista, A. O., \& Fusco, N. (2012). A influência da bidirecionalidade do sistema de escrita para a aprendizagem da leitura e da ortografia. In G. D. Germano, F. H. Pinheiro, \& S. A. Capellini (Eds.), Dificuldades de aprendizagem: Olhar multidisciplinar. (pp.119-129). Curitiba: Editora CRV.

Bell, T. (2001). Extensive reading: Speed and comprehension. The Reading Matrix, 1(1), 1-13. Retrieved from: http:/www. readingmatrix.com/articles/bell/

Braibant, J. A. (1997). Decodificação e a compreensão: dois componentes essenciais da leitura no $2^{\circ}$ ano primário. In J. Grégoire \& B. Piérart (Eds.), Avaliação dos problemas de leitura: os novos modelos teóricos e suas implicações diagnósticas (pp. 167-187). Porto Alegre: Artes Médicas.

Breznitz, Z. (2006). Fluency in reading: Synchronization of process. Mahwah, NJ: Lawrence Erlbaum.

Calhoon, M. B. (2005). Effects of peer-mediated phonological skill and reading comprehension program on reading skill acquisition for middle school students with reading disabilities. Journal of Learning Disabilities, 38(5), 424-433. doi: 10.1177/00222194050380050501

Capellini, S. A. (2004). Distúrbios de aprendizagem versus dislexia. In L. P. Ferreira, D. M. B. Lopes, \& S. C. O. Limongi (Eds.), Tratado de fonoaudiologia (1a ed., pp. 862-876). São Paulo: Editora Roca.

Capellini, S. A., Silva, A. P. C., Silva, C., \& Pinheiro, F. H. (2009). Avaliação e diagnóstico Fonoaudiológico nos distúrbios de aprendizagem e dislexias. In J. Zorzi \& S. A. Capellini (Eds.), Dislexia e outros distúrbios da leitura e escrita: Letras desafiando a aprendizagem (pp. 81-94). São José dos Campos, SP: Pulso.

Costa, M. L. G. A. (2004). Compreensão leitora e o rendimento escolar: Um estudo com alunos do $4 .^{\circ}$ ano de escolaridade (Unpublished master's thesis). Instituto de Educação e Psicologia, Universidade do Minho, Braga, Portugal. Retrieved from http://hdl.handle.net/1822/8141

Cunha, V. L. O., \& Capellini, S. A. (2009a). Desempenho de escolares de $1^{\mathrm{a}}$ a $4^{\mathrm{a}}$ série do ensino fundamental nas provas de habilidades metafonológicas e de leitura - PROHMELE. Revista da Sociedade Brasileira de Fonoaudiologia, 14(1), p. 56-68. doi: 10.1590/S1516-80342009000100011

Cunha, V. L. O., \& Capellini, S. A. (2009b). Leitura: Decodificação ou obtenção do sentido?. Revista Teias, 10(19), 1-21. Retrieved from http://www.periodicos.proped.pro.br/index.php/ revistateias/article/view/332

Cunha, V. L. O., Oliveira, A. M., \& Capellini, S. A. (2010). Avaliação e intervenção na compreensão de leitura. In S. A. Capellini, G. D. Germano, \& V. L. O. Cunha (Eds.), Transtornos de aprendizagem e transtornos da atenção (pp. 49-62). São José dos Campos, SP: Pulso.

Eckert, T. L., Ardoin, S. P., Daly, E. J., \& Martens, B. K. (2002). Improving oral reading fluency: A brief experimental analysis of combining an antecedent intervention with consequences. Journal of Applied Behavior Analysis, 35(3), 271-281. doi: 10.1901/jaba.2002.35-271

Ferreira, R. D. S. (2009). Avaliação da fluência na leitura em crianças com e sem necessidades educativas especiais: Validação de uma prova de fluência na leitura para o $2^{\circ}$ Ano do $1^{\circ}$ C.E.B. (Unpublished doctoral dissertation). Faculdade de Motricidade Humana, Universidade Técnica de Lisboa, Lisboa, Portugal. Retrieved from http://hdl.handle.net/10400.5/2142
Fuchs, L. S., Douglas Fuchs, Hosp, M. K., \& Jenkins, J. R. (2001). Oral reading fluency as an indicator of reading competence: A theoretical, empirical, and historical analysis. Scientific Studies of Reading, 5(3) 239-256. doi: 10.1207/S1532799XSSR0503 3

Giangiacomo, M. C. P. B., \& Navas, A. L. G. P. (2008). A influência da memória operacional nas habilidades de compreensão de leitura em escolares de $4^{\text {a }}$ série. Revista da Sociedade Brasileira de Fonoaudiologia, 13(1), 69-74. doi: 10.1590/ S1516-80342008000100012

Guidetti, A. A., \& Martinelli, S. C. (2007). Compreensão em leitura e desempenho em escrita de crianças do ensino fundamental. Psicologia: Revista da Vetor Editora, 8(2), 175-184. Retrieved from http://pepsic.bvsalud.org/scielo.php?script=sci arttext\&pid=S1676-73142007000200008\&lng=pt\&tlng=pt.

Kintsch, W., \& van Dijk, T. A. (1978). Toward a model of text comprehension and production. Psychological Review, 85(5), 363-394.

Manyak, P. C., \& Bauer, E. B. (2008). Explicit code and comprehension instruction for english learners. The Reading Teacher, 61(5), 432-434. doi: 10.1598/RT.61.5.9

Mcquiston, K., O'shea, D., \& Mccollin, M. (2008). Improving phonological awareness and decoding skills of high school students from diverse backgrounds. Preventing School Failure, 52(2), 67-70. doi: 10.3200/PSFL.52.2.67-72

Morais, J. (1996). A arte de ler. São Paulo: Editora da Universidade Estadual Paulista.

O'connor, R. E., Swanson, H. L., \& Geraghty, C. (2010). Improvement in reading rate under independent and difficult text levels: Influences on word and comprehension skills. Journal of Educational Psychology, 102(1), 1-19. doi: 10.1037/ a0017488

Organização para Cooperação e Desenvolvimento Econômico. (2014). PISA 2012 Results: What students know and can do - student performance in mathematics, reading and science (Volume I). Paris: OECD Publishing. Retrieved from de https:// www.oecd.org/pisa/keyfindings/pisa-2012-results-volume-I. pdf

Oliveira, K. L., Boruchotvitch, E., \& Santos, A. A. A. (2009). Leitura e desempenho escolar em alunos do ensino fundamental. In A. A. A. Santos, E. Boruchovitch, \& K. L. Oliveira (Eds.), Cloze: Um instrumento de diagnóstico e intervenção (pp.149-164). São Paulo: Casa do Psicólogo.

Oliveira, K. L., Cantalice, L. M., \& Freitas, F. A. (2009). Compreensão em leitura no ensino médio: Análise de acertos por item. In A. A. A. Santos, E. Boruchovitch, \& K. L. Oliveira (Eds.), Cloze: Um instrumento de diagnóstico e intervenção (pp.165-185). São Paulo: Casa do Psicólogo.

Oliveira, K. L., \& Santos, A. A. A. (2006). Compreensão de textos e desempenho acadêmico. Psic - Revista de Psicologia da Vetor Editora, 7(1), 19-27.

Penna, M. M. V., Sabaté, C. P., \& Burin, D. (2014). Relaciones entre decodificación, conocimiento léxico-semántico e inferencias en niños de escolaridad primaria. Interdisciplinaria, 31(2), 259-274. Retrieved from http://www.redalyc.org/articulo. oa? $\mathrm{id}=18032537005$

Perfetti, C.A. (1985). Reading ability. New York: Oxford University. Pinheiro A. M. V. (1994). Leitura e escrita: Uma abordagem cognitiva. Campinas, SP: Editorial Psy. 
Ramos, C. S. (2005). Avaliação da leitura em escolares com indicação de dificuldade de aprendizagem e escrita (Unpublished doctoral dissertation). Escola Paulista de Medicina, Universidade Federal de São Paulo, São Paulo, Brasil.

Rebelo, J. A. S. (1993). Dificuldades da leitura e da escrita em alunos do ensino básico. Rio Tinto: Edições Asa Rei.

Saine, N. L., Lekkanen, M-K., Ahonen, T., Tovlanen, A., \& Lyytinen, H. (2010). Predicting word-level reading fluency outcomes in three contrastive groups: Remedial and computer-assisted remedial reading intervention, and mainstream instruction. Learning and Individual Differences, 20(5), 402-414. doi: 10.1016/j.lindif.2010.06.004

Salgado, C. A., \& Capellini, S. A. (2008). Programa de remediação fonológica em escolares com dislexia do desenvolvimento. Pró-Fono Revista de Atualização Científica, 20(1), 31-36. doi: 10.1590/S0104-56872008000100006

Salles, J. F., \& Parente, M. A. M. P. (2002). Processos cognitivos na leitura de palavras em crianças: relações com compreensão e tempo de leitura. Psicologia: Reflexão e Crítica, 15(2), 321331. Retrieved from http://hdl.handle.net/10183/25664

Salles, J. F., \& Parente, M. A. M. P. (2004). Compreensão textual em alunos da segunda e terceira séries: uma abordagem cognitiva. Estudos de Psicologia, 9(1), 71-80. doi: 10.1590/ S1413-294X2004000100009

Sampaio, M. N., Pinheiro, F. H., \& Silva, C. (2011). Intervenção para escolares com dificuldades de aprendizagem. In S. A. Capellini, C. Silva, \& F. H. Pinheiro (Eds.), Tópicos em transtornos de aprendizagem (pp. 103-115). São José dos Campos, SP: Pulso Editorial.

Sánchez, E., García, J. R., \& Gonzalez, A. J. (2007). Can differences in the ability to recognize words cease to have an effect under certain reading conditions? Journal of Learning Disabilities, 40(4), 290-305. doi: 10.1177/00222194070400040101
Sánchez, E. (2008). Leer para aprender. In J. A. Millán. (Ed.), La lectura en España (pp. 191-208). Madri: Federación de Gremios de Editores de España.

Santos, M. T. M. (2009). Dislexia: princípios para a intervenção fonoaudiológica. In T. Barbosa et al. (Eds.), Temas em dislexia (pp. 115-122). São Paulo: Artes Médicas.

Silva C., \& Capellini, S. A. (2010). Eficácia do programa de remediação fonológica e leitura no distúrbio de aprendizagem. Pró-Fono Revista de Atualização Científica, 22(2), 131-139. doi: 10.1590/S0104-56872010000200011

Snellings, P. van der Leij, A., de Jong, P. F., Blok, H. (2009). Enhancing the reading fluency and comprehension of children with reading disabilities in an orthographically transparent language. Journal of Learning Disabilities, 42(4), 291-305. doi: $10.1177 / 0022219408331038$

Stothard, S. E. (2004). Avaliação da compreensão da leitura. In M. Snowling \& J. Stackhouse (Eds.), Dislexia, fala e linguagem (pp. 121-142). São Paulo: Artmed.

Tonelloto, J. M. F., \& Gonçalves, V. M. G. (2002). Autopercepção de escolares desatentas no ambiente escolar. Estudos de Psicologia, 19(3), 31-41. doi: 10.1590/S0103-166X2002000300004

Wise, J. C., Sevcik R. A., Morris, R. D., Lovett, M. W, \& Wolf, M. (2007). The relationship among receptive and expressive vocabulary, listening comprehension, pre-reading skills, word identification skills, and reading comprehension by children with reading disabilities. Journal of Speech, Language and Hearing Research, 50(4), 1093-1109. Retrieved from http:// www.ncbi.nlm.nih.gov/pubmed/17675607

Zorzi, J. L. (2008). Guia prático para ajudar crianças com dificuldades de aprendizagem: dislexia e outros distúrbios - Um manual de boas e saudáveis atitudes. Pinhais: Editora Melo.

Zucoloto, K. A., \& Sisto, F. F. (2002). Dificuldades de aprendizagem em escrita e compreensão em leitura. Interação em Psicologia, 6(2), 157-166. Retrieved from http://ojs.c3sl.ufpr.br/ojs2/index. $\mathrm{php} / \mathrm{psicologia/issue/view/345}$

Recebido em 12.07.2014

Primeira decisão editorial em 07.07.2015

Versão final em 06.08.2015

Aceito em 23.09.2015 